\title{
Development and Investigation of The Production Process of the Aluminum Matrix Composition Mechanically Alloyed With Single- Walled Carbon Nanotubes and the Composition Material Made of it
}

\author{
Nikolai Vetkasov ${ }^{1, *}$, Anatoliy Kapustin ${ }^{1}$, and Valeriy Sapunov ${ }^{1}$ \\ ${ }^{1}$ Ulyanovsk State Technical University, 432027, Ulyanovsk, Severnyy Venets street, 32, Russia
}

\begin{abstract}
The effect of a new suggested by authors approach for alloying of the aluminum matrix powder with carbon nanotubes is described in this paper. The manufacturing science is designed for receiving the highlyhomogenous in volume dry mixture «aluminum powder - single-walled carbon nanotubes» suitable for synthetic processing of the aluminum matrix composition by the mechanical alloying and for producing of finished aluminum matrix composition materials. The effect of the experimental investigation of production processes of the aluminum matrix composition and workpieces of finished aluminum matrix composition materials is described in this paper.
\end{abstract}

\section{Introduction}

Several researchers [1-5] testified that the achievement of high physical and mechanical properties by aluminum matrix compositions is based on the effective dispersion of carbon nanotubes (CNT) in the aluminum matrix of the composition material provided at the stage of receiving of the aluminum matrix composition (AMC) including but not limited to the method of mechanical alloying (MA). Authors of these papers fairly argue that clustering and agglomerating of CNT are critical technological issues. It is reported [6] that authors succeeded in creating and investigating of composition materials of the system «aluminum $-\mathrm{CNT}$ » which reached $184 \%$ of the composition material tensile strength reinforced with CNT. The increase is achieved by providing the homogenous distribution of CNT being grown directly at the surface of the aluminum powder by CVD method. Therefore, the critical role of the homogenous distribution and the stable bond between CNT and the matrix in providing mechanical properties of composition materials is certified. In the same survey [6] it is reported that researchers who tried to introduce $1-5$ vol. \% CNT into the structure of $\mathrm{Al}$ - composition material using the powder metallurgy method with the further spark-plasma sintering and/or the thermal deformation (the hot molding) reached the maximum increase of the tensile strength by $129 \%$ after adding of 5 vol. \% CNT.

\footnotetext{
*Corresponding author: nppwt@ulstu.ru
} 
The effect of papers prepared within the framework of the investigation of production processes of the highly-homogenous dry mixture «matrix aluminum - CNT TUBALL», production of the aluminum matrix pellet powder composition made of this mixture using the method of mechanical alloying (MA) with the high dispersion of CNT distribution in composition pellets, formation of briquettes by cold molding and hot molding of workpieces of volume composition materials $(\mathrm{CM})$ is described in this paper.

The influence of the vacuum sintering and the finishing hot extrusion on strength parameters of hot-molded CM workpieces is also estimated.

The objective of the paper is to develop and to investigate the effectiveness of the production technology of volume composition materials made of aluminum matrix composition with the high dispersion of single-walled CNT reached by reinforcing of the matrix aluminum powder by mechanical alloying with carbon nanotubes.

\section{Description of experimental investigations}

The following initial materials were used as the critical raw material:

- primary dispersed aluminum powder PAD-1 (Company standard 22436138-006-2006) made of primary aluminum A7 conforming to GOST 11069-2001 using the fraction up to 30 micron;

- single-walled carbon nanotubes TUBALL 01RW01 (Specifications 2166-00191735575-2014) with the average outer diameter not more than $3 \mathrm{~nm}$ and the full specific surface not less $300 \mathrm{~m} 2 / \mathrm{g}$;

- stearic acid (GOST 6484-96) as the drilling mud surfactant regulating the process for preventing the extremely cold-welding during MA.

Composition pellets were synthesized in accordance with the 2-stage technology developed by authors. At the first stage called the mixture normalization (MN) process because of the receipt of the highly-homogenous in volume dry mixture, the initial mixture of the matrix aluminum powder and alloying CNT are processed under low impact energy levels of mill shapes on mixture components in the author's mechanical reactor (MR). Impact energy on the mixture during the normalization is limited by the relatively low level making cold welding impossible and providing exclusively the uniform volume distribution process of CNT in the matrix powder generating the mixture with low bulk density representing compressed particles of the initial mixture aluminum powder with the developed spike surface, with particles of CNT agglomerates «captured» by it.

The mixture normalization process suggests the mandatory refinement of CNT agglomerates up to dimensions close to dimensions of primary powder pellets PAD-1. Such a uniform volume distribution of agglomerates guarantees the CNT preservation in regard to the absence of critical deteriorations at the mixture normalization stage. In addition to the above, the mandatory condition is to achieve the predominantly bound state with particles of the matrix aluminum powder deformed during the normalization process, i.e. «capturing» of the alloyed powder with the developed surface. Reaching of this condition under the uniform distribution of CNT in the aluminum powder massive is a necessary condition for the uniform distribution of CNT in the volume of finished composition pellets being formed even during MA as the result of cold-welding on the energy multiply exceeding the energy in $\mathrm{MN}$ (in these investigations fourfold).

If the mixture normalization energy is determined correctly, the bulk density will be firstly reduced and then stabilized. Stabilized mode is the normalization end indicator.

The powder mixture was produced from the matrix powder PAD-1 (97,5\% vol.), single-walled carbon nanotubes TUBALL ( $2 \%$ vol.), stearic acid ( $0,5 \%$ vol.). The mixture was firstly processed in the mixture normalization mode and then in the mechanical alloying mode in MR of the original design consisting of two internal vibration elements 
fastened in a single horizontal axis, creating vibration and power impacts on grinding ball bodies in two mutually perpendicular directions with the phase shift $30^{\circ}$. Moreover, in the working chamber of MR there are special conic elements installed turning vibration plains and forming opposite ball flows for densifying and boosting of MA. Primary mixture PAD1 and CNT were normalized and then MA in MR under the following parameters: the vibrator speed when normalizing the mixture was $200 \mathrm{rev} . / \mathrm{min}$, the duration was $160 \mathrm{~min}$; the vibrator speed when MA was $400 \mathrm{rev} . / \mathrm{min}$, the duration was $240 \mathrm{~min}$. 10-mm balls made of steel ShX15B were used as grinding bodies, mass proportion of balls and mixture was 55:1.

End criteria of the mixture normalization process were:

- the reduction of the mixture bulk density was up to $0,32 \ldots 0,38 \mathrm{~g} / \mathrm{cm} 3$ (the initial bulk density of PAD-1 was $1,08 \ldots 1,1 \mathrm{~g} / \mathrm{cm} 3$ );

- CNT agglomerate dimensions were not exceeding maximum dimensions of the primary matrix powder being alloyed;

- the process transition into the stabilized mode was characterized with the absence of changes in the mixture bulk density during not less than 20 min.

End criteria of the MA process were the following parameters of composition pellets $(\mathrm{CP})$ :

- reaching of the $\mathrm{CP}$ bulk density being different from the bulk density of the primary matrix powder for not more than $\pm 5 \%$;

- absence of free agglomerates of more than 20 microns in the massive during the control using the optical microscope.

The morphology of particles was registered during and after MN. Their condition in regard to «capturing» of nanotubes agglomerates was also estimated. The morphology and granulometric composition of the end AMC were registered after MA. AMC was controlled for the absence of free amorphous carbon which could be generated as the result of the conversion of CNT into the amorphous state when they were deteriorated in accordance with the proprietary methodology.

The morphology and particle size were estimated using the scanning electronic microscope PHENOM Pro X, the digital microscope Dino-Lite AM4112PT and the optical microscope Altami MET 6T. The X-ray diffraction analysis was carried out using the X-ray diffraction meter DRON $-3 \mathrm{M}$ in Cu-rays. The micro-hardness was measured using the micro-hardness tester Tukon 1102 Micro Vickers by the Vickers hardness test. The indenter load was $100 \mathrm{~g}$. The exposure time was $10 \mathrm{~s}$. The Rockwell hardness (HRB) of samples was measured using the hardness tester TR150M. The micro-hardness control was exercised for the estimation of the reinforcing stage distribution homogeneity in the matrix. The dispersion of $\pm 10 \%$ from the average value in the series of measurements was considered as allowable. HRB control was exercised for the estimation of the CM sample hardness. Cold molding compaction was carried out using the press PB6330 with the pressure up to $100 \mathrm{t}$, special tooling designed and produced by authors and additional equipment. Compacting was carried out by 2 -sided pressing of $\mathrm{CP}$ under the pressure of $600 \mathrm{MPa}$ at $20 \ldots 25^{\circ} \mathrm{C}$ into cakes of $39,2 \mathrm{~mm}$ in diameter.

Cakes produced using the cold molding technology were preliminary heated in the press mold to $550{ }^{\circ} \mathrm{C}$, soaked at the same temperature for degassing during $5 \mathrm{~min}$ and then hotmolded under the pressure of $550 \mathrm{MPa}$ at $540{ }^{\circ} \mathrm{C}$ at the load exposure for $5 \mathrm{~min}$. As the result, CM workpieces in the form of tablets of $39,5 \mathrm{~mm}$ in diameter were produced.

The vacuum sintering of CM workpieces was carried out in the tubular furnace PT-1200 at $550{ }^{\circ} \mathrm{C}$ during $150 \mathrm{~min}$.

To investigate the feasibility and the practicability of applying the hot extrusion of CM workpieces produced by the hot-molding method with the further vacuum sintering, workpieces were hot-extruded at $460 \ldots 470{ }^{\circ} \mathrm{C}$ with the extrusion coefficient $\mathrm{k}=24$ into the 
rod of $8 \mathrm{~mm}$ in diameter. Cakes were hot-molded and workpieces were hot-extruded (using the reverse extrusion method) using the original tooling and the press PB6330. Results were estimated by changing of the tensile strength of workpieces produced from hot-molded workpieces. The extrusion impact on $\mathrm{CM}$ hardness characteristics was estimated taking into consideration the fact that when extruding material impacts on the CM workpiece structure already being hot-molded can take place. These impacts can lead to the additional shift and sliding of particles relative to each other, boundary sliding of particles when they are volume-deformed and the deterioration of nanotubes when they are critically bended and affected by cut side-forces [7-8].

Technology efficiency indicators in large were density and hardness (in HRB units) of $\mathrm{CM}$ workpieces and also the tensile strength value of samples produced from $\mathrm{CM}$ workpieces. The tensile strength of samples was measured in accordance with GOST149784 (ISO 6892084) «Metals. Tensile test methods».

\section{Results and discussion}

At the AMC synthesis stage series of MA processes with the preliminary mixture normalization were carried out.

During the normalization process within $160 \mathrm{~min}$ the mixture bulk density was decreasing till the stationary value $0,34 \ldots 0,36 \mathrm{~g} / \mathrm{cm}^{3}$ (Fig. 1) suggesting the right choice of the low-energy impact level with excluding the cold-welding process being characterized with CP forming and increasing of their bulk weight.

After the mixture normalization during MA within 240 min volume CP were formed. Their sizes were increasing and their structures were firming with their bulk density increase. The bulk weight was increasing from the stationary minimal weight being reached at the mixture normalization stage to the stationary value close to the bulk weight of the primary matrix powder PAD-1 (Fug. 1).

See the graph of the mixture bulk weight variance in the Fig. 1.

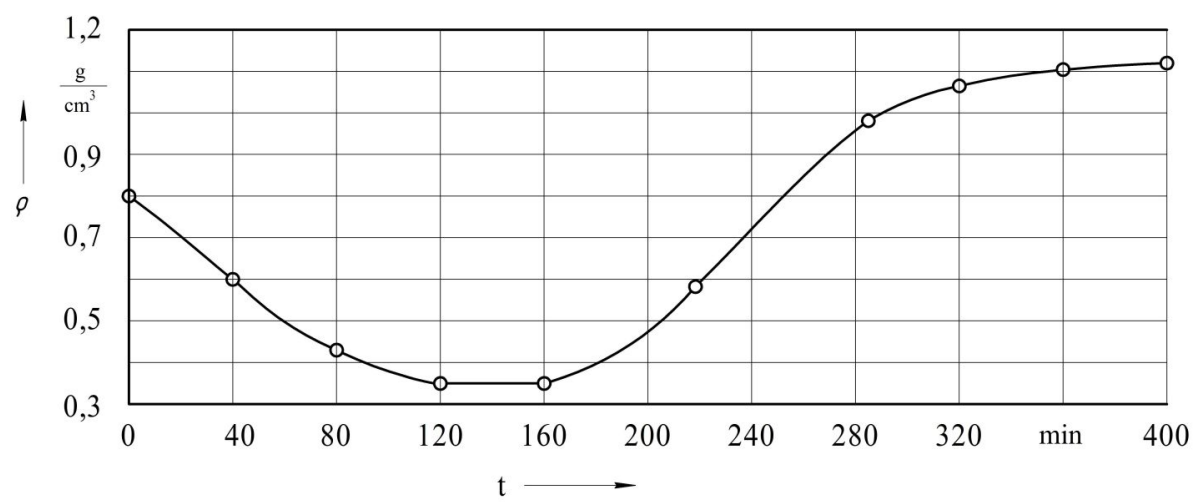

Fig. 1. The bulk density variance during the sequential mixture normalization process and the composition pellets production by mechanical alloying

Table 1. Granulometric composition of composition pellets after MA processes

\begin{tabular}{|c|c|c|c|}
\hline \multicolumn{4}{|c|}{ Granulometric composition, vol. \% } \\
\hline $0-63$ microns & $63-140$ microns & $140-700$ microns & $>700$ microns \\
\hline 20,5 & 41,9 & 37,5 & 0,1 \\
\hline
\end{tabular}

The particle morphology analysis of the normalized mixture proved its conformity with mixture normalization quality criteria. Capturing of CNT agglomerates by the developed 
surface of deformed matrix aluminum particles was observed. Agglomerates were captured by both the periphery and the surface of deformed aluminum particles, their multiple ruptures and buckles.

The morphology analysis of AMC produced after the finished MA process in regard to the state transformation of agglomerates formed as the result of CNT MN showed their total disappearance. It means that they were absorbed by the surface and the massive of pellets both investigated and their finer components. It is apparent that the surface of alloyed pellets gained on the expressed dark-grey color contracting with the silver color of the pure matrix aluminum powder after MN and MA in the same modes (Fig. 2). This suggests the presence of CNT directly at the CP surface layer generated by surfaces of composing fine pellets. It is obvious that this is also related to internal division surfaces among fine pellets composing observed CP during MA and as the result of cold-welding [910]. This fact determines the high volume homogeneity of CNT distribution both in CP massive and the end CM.

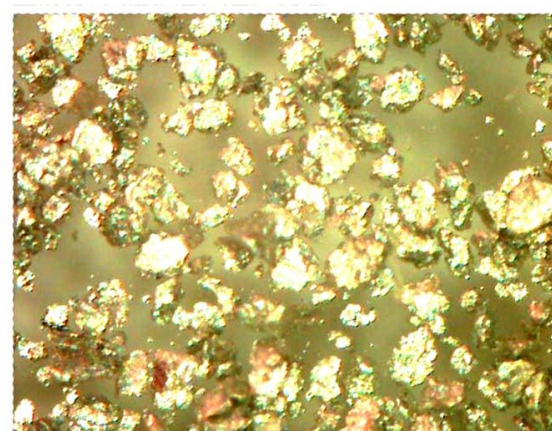

a)

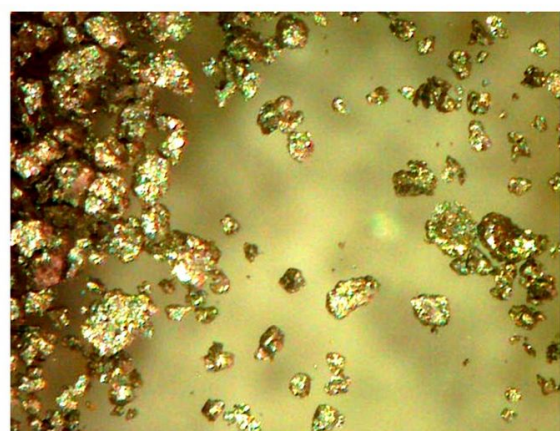

b)

Fig. 2. Appearance of pellets (power is $\times 200$ ): a - pellets of pure aluminum matrix powder; $b$ composition pellets with CNT

Coloring of $\mathrm{CP}$ surface generated by surfaces of consisting finer pellets, nanotubes tightly bound with the matrix material is proved, firstly, by verifying the absence of coloring of $\mathrm{CP}$ surface by free carbon which could be generated as the result of the nanotubes conversion into the amorphous state by the procedure and, secondly, by verifying the absence of coloring as the result of the generation of CNT layer, free or loosely bound with the surface of the matrix material at CP surface, using the ultrasonic processing of CP by the ultrasonic disperser IL 100-6/4 in isopropyl alcohol with the generation of the persistent dispersion if there are free nanotubes [11-12].

In the following stages produced AMC were firstly compacted by cold-molding. As the result cakes in the range $2,4 \ldots 2,42 \mathrm{~g} / \mathrm{cm}^{3}$ were produced. One group of cakes was further hot-molded. Another group of cakes was firstly hot-molded and then vacuum-sintered. A part of workpieces after vacuum sintering were hot-extruded. Strength parameters were measured for every group of workpieces and produced samples.

$\mathrm{X}$-ray diffraction analysis showed that during $\mathrm{MN}$ with further MA average dimensions of crystallites were decreased from 93,3 nm (primary powder) to 36,2 nm (alloyed pellets). After hot molding and vacuum sintering average dimensions of crystallites were $36,8 \mathrm{~nm}$. This indicates that during hot forming of $\mathrm{CM}$ dimensions of crystallites in the composition are not materially affected. The X-ray diffraction pattern of samples with CNT (Fig. 3) shows the absence of aluminum carbide reflexes after hot molding and vacuum sintering. This certifies the preservation of nanotubes in CM massive (the absence or light conversion into the amorphous state). 


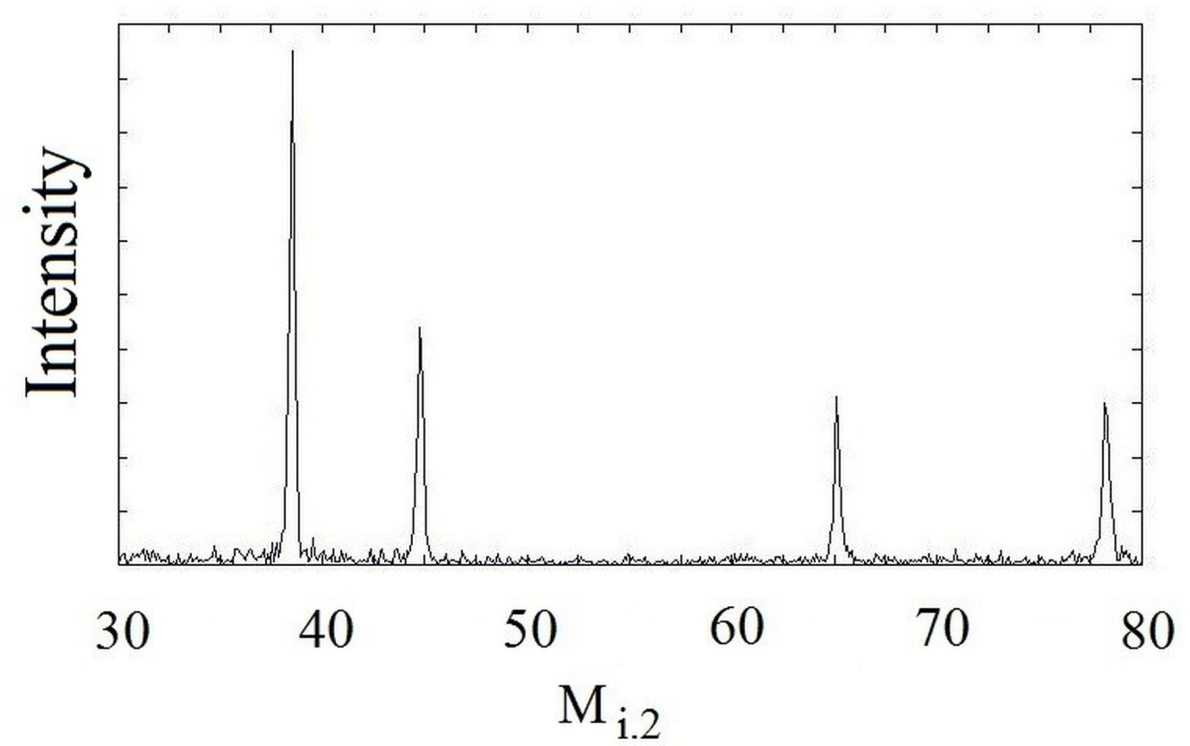

Fig. 3. X-ray diffraction pattern of samples after hot molding and vacuum sintering

Data about strength parameters of CM workpieces produced as the result of hot molding and after vacuum sintering are listed in the Table 2 .

Table 2. Aggregated parameters of CM workpieces and samples produced as the result of hot molding, before and after vacuum sintering

\begin{tabular}{|c|c|c|c|c|}
\hline $\begin{array}{c}\text { Workpiece } \\
\text { receive } \\
\text { operation }\end{array}$ & $\begin{array}{c}\text { Sample } \\
\text { density, } \mathrm{g} / \mathrm{cm}^{3}\end{array}$ & $\begin{array}{c}\text { Micro-hardness, } \\
\text { MPa }\end{array}$ & $\begin{array}{c}\text { Hardness } \\
\text { HRB }\end{array}$ & $\begin{array}{c}\text { Tensile strength } \sigma \mathrm{v}, \\
\text { MPa }\end{array}$ \\
\hline Hot molding & 2,69 & 1020 & 84 & 210 \\
\hline $\begin{array}{c}\text { Vacuum } \\
\text { sintering }\end{array}$ & 2,7 & 910 & 82 & 249 \\
\hline
\end{tabular}

The tensile strength of $\mathrm{CM}$ samples and workpieces after produced as the result of vacuum sintering, after hot extruding into the rod was $265 \mathrm{MPa}$.

The tensile strength of CM samples after vacuum sintering is increased by $10-15 \%$. In addition to the above, the hot extrusion after sintering additionally increases the tensile strength (about 5\%). It means that the extrusion in applied extrusion modes of $\mathrm{CM}$ compacts does not lead to the degradation of material strength properties (there are no critical deteriorations of CNT in CM).

The light decrease of the micro-hardness and HRB hardness after sintering is explained by the diffusion bonding of pellets during sintering in the low-melting phase - aluminum (characteristic of sintering of powder compositions consisting of 2 mutually insoluble components) [13-15].

\section{Conclusions}

1. New achievement principle and specific method for producing of the homogenous CNT dispersion for the further production of the aluminum matrix composition with the highly-homogenous CNT distribution in the matrix material during the mixture normalization were designed and implemented.

2. Basics of the production technology of aluminum matrix compositions reinforced 
with CNT giving an opportunity to resolve the issue of producing CM with the highlyhomogenous CNT distribution in the massive and achieving the high tensile strength were designed.

3. It was shown that the application of designed mechanical alloying technologies of the aluminum powder CNT with the preliminary mixture normalization and hot molding of the produced composition with further vacuum sintering provided the increase of the tensile strength of the matrix aluminum by not less than fourfold.

4. Core parameters and requirements to hot-molding and vacuum-sintering modes when producing volume aluminum matrix CM were determined.

The work was executed and this paper was drawn up with the financial support of the Russian Foundation for Basic Research (the project 16-43-732150 p_oфu_m).

\section{References}

1. Anantha Prasad M.G., Bandekar N., J. of Materials Science and Chemical Engineering 03, 3 (2015)

2. Ostovan F., Matori K.A., Toozandehjani M., Oskoueian A., Yusoff H.M., Yunus R., Ariff A.M., Quah H.J., Lim W.F., Materials Chemistry and Physics 166, 160 (2015)

3. Zhu X., Zhao Y.G., Wu M., Wang H.Y., Jiang Q.C., Materials 3, 3 (2016)

4. Choi H., Shin J., Min B., Park J., Bae D., J. of Materials Research 24, 2610 (2011)

5. Yang C., Zong Y., Zheng Z., Shan D., Materials Characterization 96, 84 (2014)

6. A. Agarwal, S.R. Bakshi, D. Lahiri, International Materials Reviews 1, 41 (2010)

7. Raviathul B.M., Srivastava V.C., Mukhopadhyay N.K., Materials \& Design 64, 542 (2014)

8. Ci L., Ryu Z., Jin P.N.Y., Rühle M., Acta. Mater 54, 5367 (2006)

9. Hassan M.T.Z., Esawi A.M.K., Metwalli S., J. of Alloys and Compounds 607, 215 (2014)

10. Yoo S.J., Han S.H., Kim W.J., Scr. Mater. 68, 711 (2013)

11. Wang L., Choi H., Myoung J.M., Lee W., Carbon 47, 3427 (2009)

12. Nayan N., Murty S.V.S.N., Sharma S.C., Kumar K.S., Sinha P.P., Materials Characterization 62, 1087 (2011)

13. Kukovecz A., Kanyo T., Konya Z., Kiricsi I., Carbon 43, 994 (2005)

14. Nam H.R., Kim Y.J., Yang S.S., Ahn J.H., J. of Nanoscience and Nanotechnology 14, 9103 (2014)

15. Narayanan S.S., Manickavasaham G., Moorthy S., IOSR J. of Mechanical and Civil Engineering 12, 30 (2015) 